\title{
Human Umbilical Cord Mesenchymal Stem Cells Improve the Necrosis and Osteocyte Apoptosis in Gucocorticoid-Induced Osteonecrosis of the Femoral Head Model through Reducing the Macrophage Polarization
}

\author{
Gang Tian ${ }^{1, *}$, Chuanjie $\mathrm{Liu}^{2,3, *}$, Qi Gong ${ }^{3}$, Zhiping $\mathrm{Yu}^{4}$, Haitao Wang ${ }^{5}$, Daoqiang Zhang ${ }^{3}$, Haibo Cong ${ }^{6}$ \\ ${ }^{1}$ Department of Orthopedics, Weihai Central Hospital Affiliated to Qingdao University \& Qingdao University, Weihai, China \\ ${ }^{2}$ Xinxiang Medical University, Xinxiang, China \\ ${ }^{3}$ Weihai Key Laboratory of Autoimmunity \& Central Laboratory of Weihai Central Hospital, Weihai, China \\ ${ }_{5}^{4}$ Department of Sports Medicine, Weihai Central Hospital Affiliated to Qingdao University, Weihai, China \\ ${ }^{5}$ Department of Trauma Surgery, Weihai Central Hospital Affiliated to Qingdao University, Weihai, China \\ ${ }^{6}$ Department of Orthopedics, Weihai Central Hospital Affiliated to Qingdao University \& Weihai Key Laboratory of Autoimmunity, \\ Weihai, China
}

\begin{abstract}
Background and Objectives: Apoptosis is an outstanding determinant of glucocorticoid (GC)-induced osteonecrosis of the femoral head (ONFH). Human umbilical cord mesenchymal stem cells (hUC-MSCs) have been demonstrated to be associated with apoptosis in diseases models. However, the role of hUC-MSCs in GC-induced ONFH via regulating apoptosis still needs further study.

Methods and Results: In the present study, a GC-induced ONFH model was built in vivo through a consecutive injection with lipopolysaccharide (LPS) and methylprednisolone. The necrosis and apoptosis of the femoral head was evaluated by histological and Terminal-deoxynucleoitidyl Transferase Mediated Nick End Labeling (TUNEL) assay. The level of collagen and TRAP positive cells were determined by Masson and TRAP staining, respectively. Ml macrophage polarization was assessed using immunofluorescence assay. The level of proinflammatory cytokines including tumor necrosis factor (TNF)- $\alpha$, Interleukin (IL)- $1 \beta$ and IL-6 of femoral head was determined by enzyme-linked immunosorbent assay (ELISA) kits. The protein expression of AKT, mTOR, p-AKT and p-mTOR was detected using western blot assay. The results showed that hUC-MSCs treatment prominently promoted the GC-induced the decrease of the collagen level and the increase of TRAP positive cells. Besides, hUC-MSCs treatment decreased necrosis and apoptosis, macrophage polarization, the level of TNF- $\alpha$, IL-1 $\beta$ and IL-6, the protein expression of p-AKT and p-mTOR, and the radio of p-AKT to AKT and p-mTOR to mTOR of femoral head in vivo.

Conclusions: Therefore, the present study revealed that hUC-MSCs improved the necrosis and osteocyte apoptosis in GC-induced ONFH model through reducing the macrophage polarization, which was associated with the inhibition of $\mathrm{AKT} / \mathrm{mTOR}$ signaling pathway.
\end{abstract}

Keywords: Osteonecrosis of the femoral head (ONFH), Glucocorticoid, Human umbilical cord mesenchymal stem cells, Apoptosis, Macrophage polarization

Received: June 28, 2021, Revised: October 1, 2021, Accepted: October 25, 2021, Published online: December 31, 2021

Correspondence to Daoqiang Zhang

Weihai Key Laboratory of Autoimmunity \& Central Laboratory of Weihai Central Hospital, 3 Midong Road West, Wendeng District, Weihai 264400, China

Tel: +86-18306305073, Fax: +86-0631-3806831, E-mail: zhdqwd@163.com

Co-Correspondence to Haibo Cong

Department of Orthopedics, Weihai Central Hospital Affiliated to Qingdao University \& Weihai Key Laboratory of Autoimmunity, 3 Midong Road West, Wendeng District, Weihai 264400, China

Tel: +86-13906318868, Fax: +86-0631-3806830, E-mail: haibocong@163.com

*These authors contributed equally to this work.

(9) This is an open-access article distributed under the terms of the Creative Commons Attribution Non-Commercial License (http://creativecommons.org/ licenses/by-nc/4.0/), which permits unrestricted non-commercial use, distribution, and reproduction in any medium, provided the original work is properly cited. Copyright (c) 2022 by the Korean Society for Stem Cell Research 


\section{Introduction}

Osteonecrosis of the femoral head (ONFH) is a chronic and disabling disease that mainly comprises traumatic and non-traumatic ONFH (1). Use of glucocorticoids (GC) is the main reason for non-traumatic ONFH (2). The majority of ONFH patients receive the long-term GC therapy (3). Moreover, patients with GC-induced ONFH generally obtain surgical treatment owing to the lack of effective drugs. Thus, it is completely important to explore the potential molecular mechanism involved in GC-induced ONFH contributing to the underlying therapeutic targets and strategies of ONFH.

Macrophages, as a type of immune cells, play an important role in both innate and adaptive immune response that participate in the occurrence and development of various inflammatory and immune-related diseases (4). Macrophages are classified into M1 and M2 phenotypes according to the surface leukocyte differentiation antigen (cluster of differentiation, CD) and its effects (5). M1 phenotype is involved in the host's defense against pathogens (6) that can secrete a large number of pro-inflammatory cytokines such as TNF- $\alpha$, IL-1 $\beta$ and IL-6. M2 phenotype secretes IL-10 and TGF- $\beta$, which promotes tissue repair and inflammation subsides to exert the anti-inflammatory function (7). The great mass of resident femoral head macrophages can polarize to the $\mathrm{Ml}$ phenotype to cause the increase of the level of the pro-inflammatory cytokines including IL-1 $\beta$ and TNF- $\alpha$, which facilitates the apoptosis of osteocyte $(8,9)$. Apoptosis of osteocytes is the primary cause of bone cell death in patients with GC-induced ONFH accompanied with a prominent decrease of osteocyte viability in the femoral head $(10,11)$. Therefore, the macrophage polarization may be tightly associated with the occurrence and development of GC-induced ONFH.

Human umbilical cord mesenchymal stem cells (HUCMSCs) are a category of mesenchymal stem cells that can differentiate into diverse cells. Thus, hUC-MSCs have been applied for a variety of diseases, such as cerebral ischemia (12), spinal cord injury (13) and traumatic brain injury (14). Recent studies show that MSCs can significantly increase the infiltration of M2 macrophages in the area of myocardial infarction, reduce fibrosis, increase angiogenesis, and improve heart function (15). Moreover, MSCs have been reported to promote the conversion of M1 to M2 in several extracardiac organs including bone marrow and spleen (15). Besides, the MSCs transplantation has shown the potentially clinical application in the treatment of inflammatory diseases (16). Furthermore, hUC-MSCs relieve inflammatory response through the transition from both intracardiac and extracardiac M1 to M2 phenotype to reduce the level of inflammatory factor, which ameliorates cardiac function and protects the infarcted myocardium in acute myocardial infarction model (17). However, whether hUC-MSCs inhibit the GC-induced $\mathrm{ONFH}$ via promoting the conversion of $\mathrm{M} 1$ to $\mathrm{M} 2$ is still unclear.

In the present study, the role of hUC-MSCs in the GC-induced ONFH was investigated. The results showed that hUC-MSCs could mitigate the inflammatory cell polarization through promoting the transition of $M 1$ to $M 2$ phenotype, which reduces necrosis and osteocyte apoptosis in a GC-induced ONFH rat model. Mechanically, the protective role of hUC-MSCs in the GC-induced ONFH was involved in the $\mathrm{AKT} / \mathrm{mTOR}$ signaling pathway.

\section{Materials and Methods}

\section{Preparation of hUC-MSCs}

hUC-MSCs were separated from the umbilical cord samples obtained from normal term cesarean section neonates. The mother and child with any infectious diseases and family genetic diseases were excluded, and informed consent was obtained from the mother and the family. The study was authorized by the Board and Ethics Committee of Weihai Central Hospital Affiliated to Qingdao University. Cells were passed until the confluent of cells reached approximately $80 \%$.

\section{Animal model and experimental groups in vivo}

18 healthy female Sprague-Dawley (SD) rats (eight weeks, $300 \pm 20 \mathrm{~g}$ ) were divided into three groups in random $(n=6)$ : control group, model group and hUC-MSCs group. All the experiments were performed with the approval of ethical standards as decided by the ethical committee of West China Hospital of Sichuan University (20211302A). Rats were intraperitoneally injected with lipopolysaccharide (LPS) (40 $\mu \mathrm{g} / \mathrm{kg} / \mathrm{d})$ for two consecutive days, then intramuscularly injected with methylprednisolone $(40 \mathrm{mg} / \mathrm{kg} / \mathrm{d})$ from day 3 to day 7 , and continuously fed about 12 weeks to build the ONFH model. Rats in the hUC-MSCs group were injected with $0.2 \mathrm{ml}$ hUCMSCs (about $1 \times 10^{6}$ cells) via the tail vein once a week for a total of 6 weeks, whereas rats in control and model group were injected with $0.2 \mathrm{ml}$ of phosphate buffer saline (PBS) (Zhongshan Golden Bridge Biological Technology, Beijing, China) via the tail vein. Rats were sacrificed six weeks after the last injection. After the rats were intraperitoneally anesthetized with sodium pentobarbital (40 $\mathrm{mg} / \mathrm{kg}$ ), peripheral blood was taken, and serum was iso- 
lated and stored at $-80^{\circ} \mathrm{C}$ for further assays. The femoral head tissues were fleetly removed for histological and western blot analysis.

\section{H\&E, Masson and TRAP staining assays}

The femoral heads were separated, fixed, decalcified, embedded and cut into sections. $5 \mu \mathrm{m}$ sections were stained with hematoxylin and eosin $(\mathrm{H} \& \mathrm{E})$, Masson and TRAP. Pictures were obtained under a microscope (DMI, LEICA, Germany).

\section{TUNEL staining}

Apoptosis in the femoral head tissues was determined by TUNEL staining. In brief, the paraffin sections $(5 \mu \mathrm{m})$ were performed using TUNEL apoptosis detection kit (49330900, Roche Applied Science, Switzerland) according to the manufacturer's instructions. Images were analyzed with a light microscope (DMI1, LEICA). Apoptotic index was exhibited as the ratio of the number of TUNEL positive cells to the total number of cells.

\section{Immunofluorescence assay}

The femoral head tissues were separated and rapidly frozen in OCT using liquid nitrogen. The sections $(5 \mu \mathrm{m})$ were acquired by a cryostat, and then mounted on microscope slides (Fisherbrand Superfros Plus, Fisher Scientific). The sections were fixed with 4\% paraformaldehyde for 15 min. Subsequently, the sections were blocked with goat serum, and then incubated with the primary antibody (1 : 100, Rabbit monoclonal antibodies CD86, \#48763, Signalway, Maryland, USA; $1: 100$, Rabbit monoclonal antibodies CD206, ab64693, Abcam, Cambridge, UK; 1 : 100, Rat monoclonal antibodies F4/80, ab16911, Abcam) at $4{ }^{\circ} \mathrm{C}$ overnight. After being washed with PBS thrice, the sections were incubated with Cy3-labeled goat anti-rat IgG (GB21302; 1 : 100, Servicebio, Wuhan, China) and FITClabeled goat anti-rabbit IgG (GB22303, 1 : 100; Servicebio) for $60 \mathrm{~min}$ at $37^{\circ} \mathrm{C}$ respectively. The nuclei were stained with DAPI (ZLI-9557, Zhongshan Golden Bridge Biological Technology, Beijing, China). The proportion of M1 and M2 was assessed with the mean optical density that was determined using the ImageJ analysis software.

\section{Enzyme-linked immunosorbent assay (ELISA)}

The levels of TNF- $\alpha$, IL- $1 \beta$ and IL- 6 in the femoral heads were determined using Rat TNF- $\alpha$ ELISA KIT (ZC-37624, ZCi Bio, Shanghai, China), Rat IL-1 $\beta$ ELISA Kit (ZC-36391, ZCi Bio), and Rat IL-6 ELISA KIT (ZC-36404, ZCi Bio) according to the manufacturer's protocol. The absorbance of wells was determined with a microplate reader (spectra max PLUS 384, Molecular Devices, California, USA) at $450 \mathrm{~nm}$ wavelength to analyze the sample concentration.

\section{Western blot assay}

Protein samples from femoral head tissues were obtained using a Total Protein Extraction Kit (BC3711, Solarbio). Then, the protein concentration was determined by a Protein Assay kit (Beyotime, Shanghai, China). Protein samples were separated by $10 \%$ SDS-PAGE gel and electrically transferred to PVDF membranes (Millipore, Billerica, MA, USA). The membranes were hatched with the primary antibodies at $4{ }^{\circ} \mathrm{C}$ overnight after being blocked with $3 \%$ bovine serum albumin (BSA, Sangon Biotech, Shanghai, China) for $1 \mathrm{~h}$ at room temperature. The primary antibodies were listed as followed: rabbit anti-AKT (ab179463, 1 : 10,000); rabbit anti-p-AKT (ab81283, $1: 1,000)$; rabbit anti-mTOR (ab2732; $1: 10,000)$; rabbit anti-p-mTOR (ab63552; $1: 1,000)$; rabbit anti- $\beta$-actin (ab8227; 1 : 1,000) (Abcam, Cambridge, UK). The membranes were incubated with goat-anti-rabbit $\operatorname{IgG}(\mathrm{H}+\mathrm{L})-\mathrm{HRP}$ (1 : 10,000, ab6721, Abcam) for $1 \mathrm{~h}$ at room temperature after washing with TBST thrice. Protein bands were visualized with an Electrochemilluminescence (ECL) chemiluminescence kit (WBULS0500; EMD Millipore) and the bands intensity were analyzed with Image-Pro Plus 6.0 software.

\section{Statistical analysis}

Data were shown as the means \pm standard deviation. Differences among multiple groups were analyzed using one-way analysis of variance and Duncan's test using the SPSS 20.0 package (SPSS Inc. Chicago, IL, USA). The differences were regarded as statistically non-significant and significant when $\mathrm{p}>0.05$ and $\mathrm{p}<0.05$, respectively.

\section{Results}

\section{hUC-MSCs mitigated osteonecrosis and apoptosis in a GC-induced ONFH rat model}

Flow cytometry analysis revealed that the passage three of hUC-MSCs had a prominent expression of CD29, CD44, CD73, CD90 and CD105, and almost did not express CD14, CD34, CD45 and HLA-DR (Supplementary Fig. S1). The H\&E staining showed the necrosis of the femoral head chondrocytes, the disappearance of the columnar arrangement, the collapse of the trabeculae of subchondral bones and an increase of empty bone lacunas number in the model group compared with that in the control group. However, these pathological characteristics were alleviated in the hUC-MSCs group as indicated with 
a less necrosis of the femoral head chondrocytes, a less collapse of the trabeculae of subchondral bones and a decrease of empty bone lacunas number compared with that in the model group (Fig. 1A). TUNEL staining revealed that the apoptosis was dramatically increased in the model group compared with that in the control group, which was observably antagonized with hUC-MSCs treatment (Fig. 1B). Thus, the results indicated that hUC-MSCs inhibited osteocyte apoptosis. Masson and TRAP staining revealed that the hUC-MSCs treatment can significantly increase the collagen level and decrease the TRAP positive cells in the GC-induced femoral head (Fig. 1C and 1D). These results suggested that a GC-induced ONFH rat model was successfully established, and hUC-MSCs could alleviate osteonecrosis of the GC-induced ONFH rat model.

\section{hUC-MSCs decreased M1 polarization in femoral heads}

Immunofluorescence results exhibited that the percentage of CD86+ F4/80+cells/ F4/80+cells and the percentage of $\mathrm{F} 4 / 80+$ cells/ DAPI cells in model group was prominently enhanced compared with that in control group, which was significantly reversed with hUC-MSCs treatment (Fig. 2A). On the contrary, the percentage of $\mathrm{CD} 206+\mathrm{F} 4 / 80+$ cells/ F4/80 + cells in model group was notably reduced compared with that in control group, which was observably inverted with hUC-MSCs treatment (Fig. 2B). These findings suggested that hUC-MSCs decreased M1 polarization in femoral heads.

\section{hUC-MSCs reduced the level of proinflammatory cytokines in the femoral heads}

Moreover, the level of proinflammatory cytokines including TNF- $\alpha$, IL- $1 \beta$ and IL- 6 in the femoral heads was determined by ELISA kits. The results showed that all the secretion of TNF- $\alpha$, IL-1 $\beta$ and IL- 6 was significantly elevated in the femoral heads of GC-induced ONFH rat model. However, the level of TNF- $\alpha$, IL- $1 \beta$ and IL- 6 was notably decreased in the GC-induced ONFH rat model treated with hUC-MSCs (Fig. 3). Therefore, the results indicated that the level of proinflammatory cytokines was
A
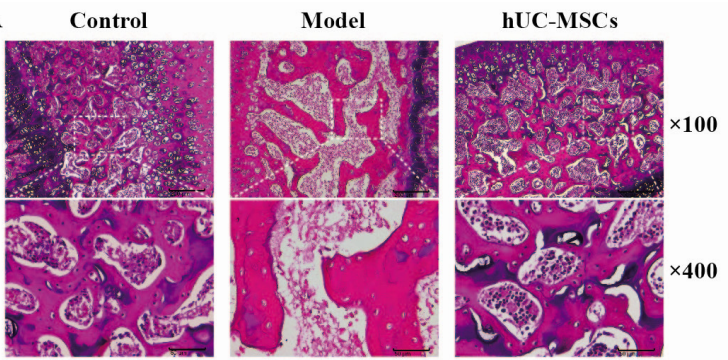

C

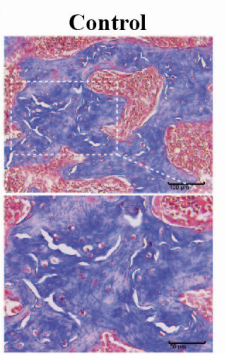

Model
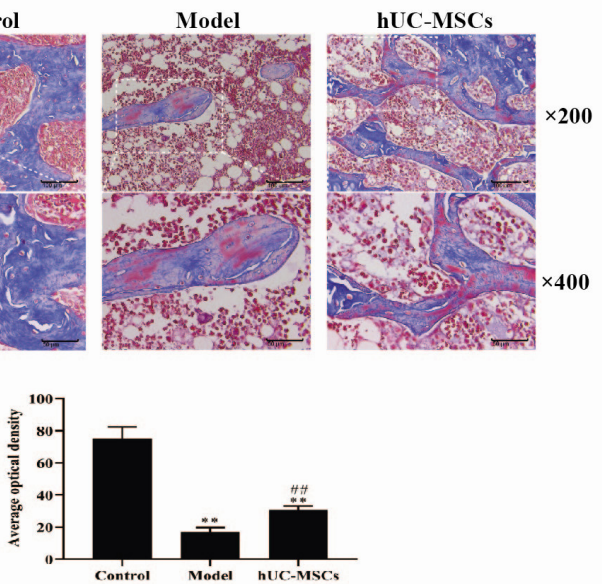
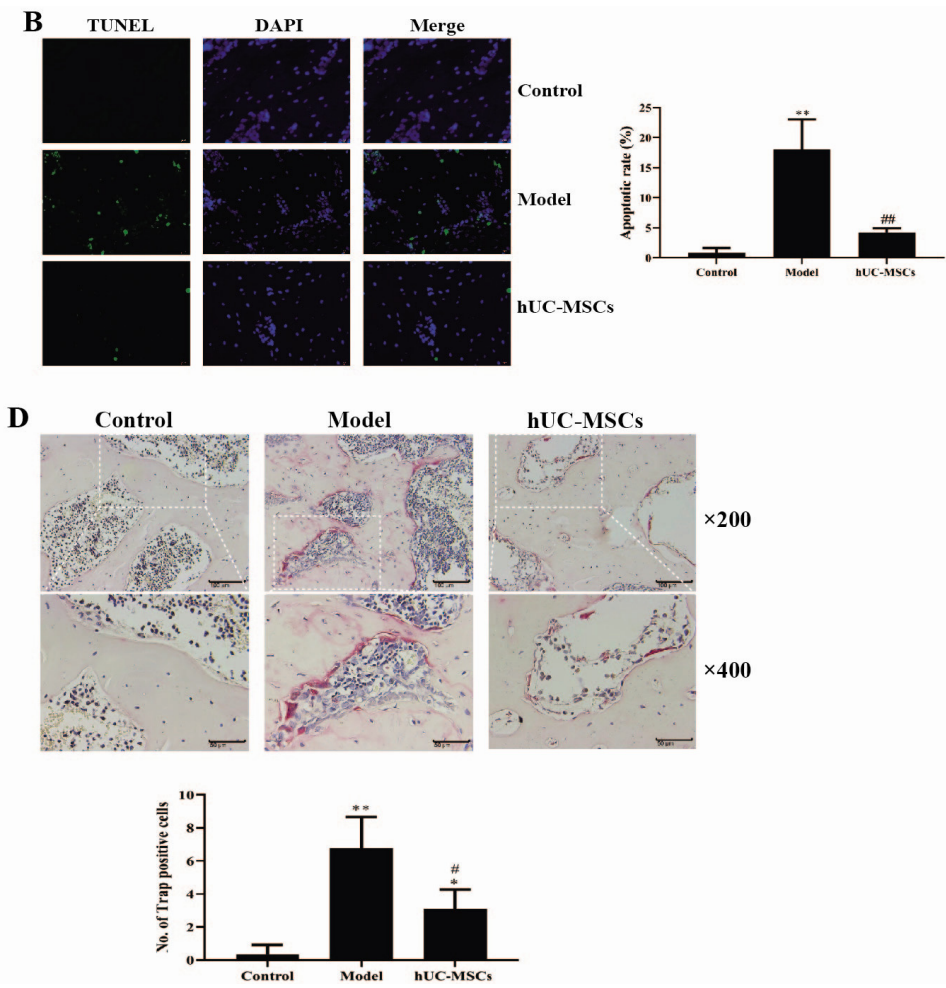

Fig. 1. hUC-MSCs mitigated osteonecrosis and apoptosis in a GC-induced ONFH rat model. (A) Histological analysis was determined by H\&E staining (magnification, $\times 100$ or $\times 400$ ). Scale bar: $50 \mu \mathrm{m}$. (B) The apoptosis rate of femoral head tissues was determined by TUNEL (magnification, $\times 400$ ). Scale bar: $20 \mu \mathrm{m}$. (C) The collagen level of femoral head was determined by Masson staining (magnification, $\times 400$ ). Scale bar: $20 \mu \mathrm{m}$. (D) The Number of osteoclasts was detected by TRAP staining (magnification, $\times 400$ ). Scale bar: $20 \mu \mathrm{m}$. The means \pm SD of six independent samples were shown. ${ }^{*} \mathrm{p}<0.05$ and ${ }^{* *} \mathrm{p}<0.01$, compared with control group; ${ }^{\#} \mathrm{p}<0.05$ and ${ }^{\# \#} \mathrm{p}<0.01$, compared with model group. 


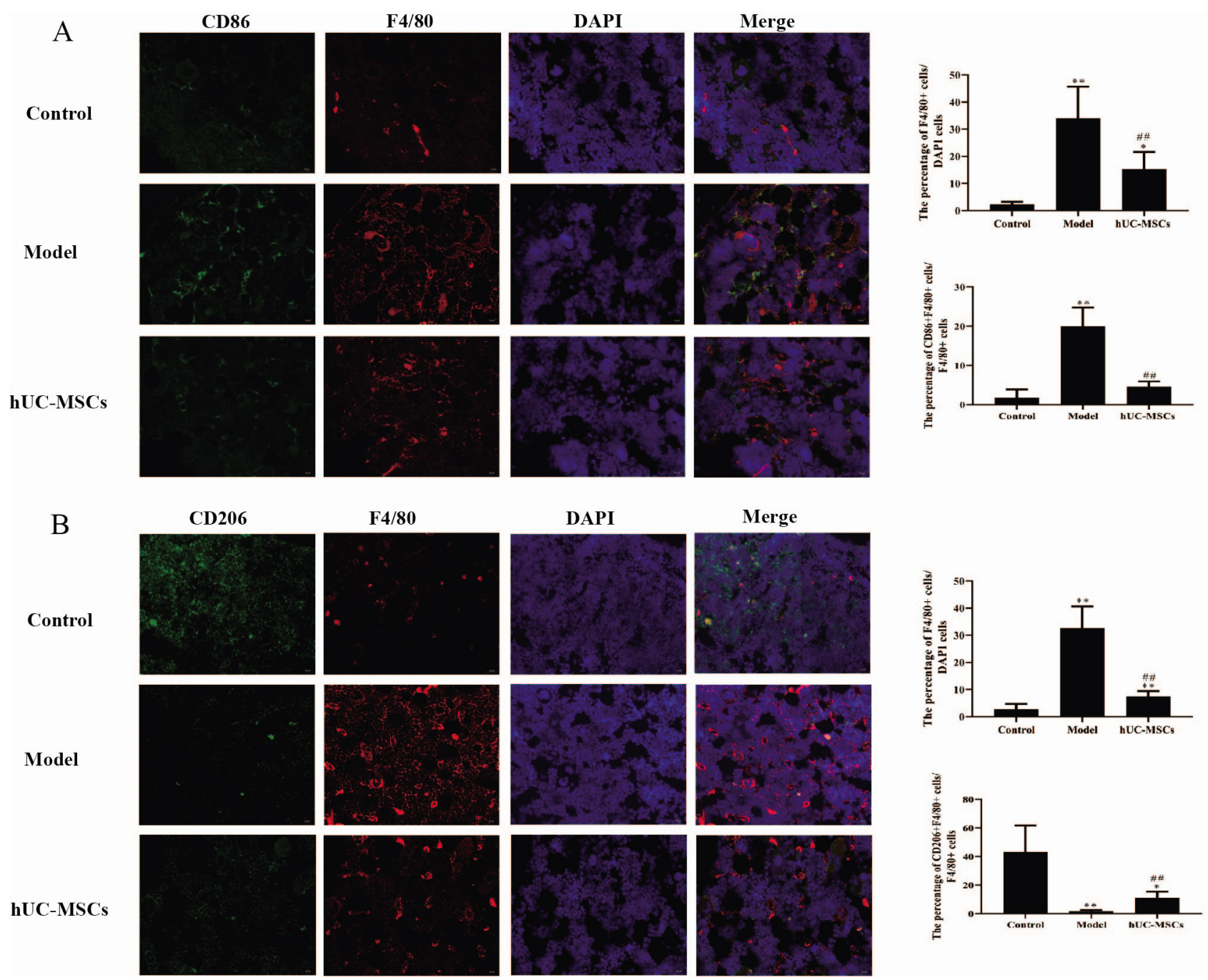

Fig. 2. hUC-MSCs reduced M1 polarization in femoral heads. (A) The CD86 and F4/80 of macrophages were stained using immunofluorescence assay (magnification, x400). Scale bar: $20 \mu \mathrm{m}$. (B) The CD206 and F4/80 of macrophages were stained using immunofluorescence assay (magnification, $\mathrm{x} 400$ ). Scale bar: $20 \mu \mathrm{m}$. The means \pm SD of six independent samples were shown. ${ }^{*} \mathrm{p}<0.05$ and ${ }^{*} \mathrm{p}<$ 0.01 , compared with control group; ${ }^{\#} \mathrm{p}<0.01$, compared with model group.

A

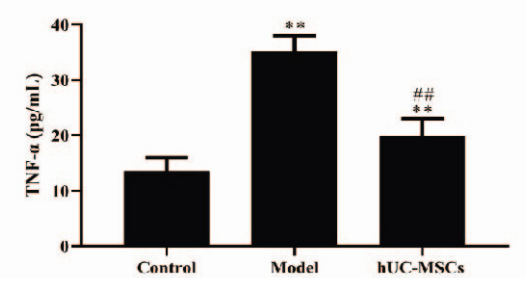

B

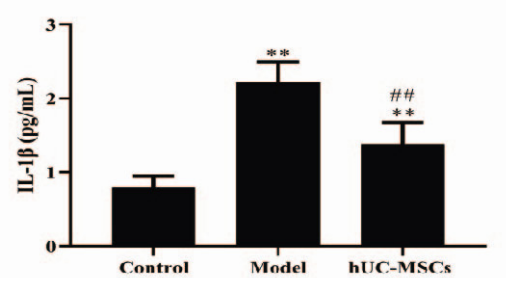

$\mathrm{C}$

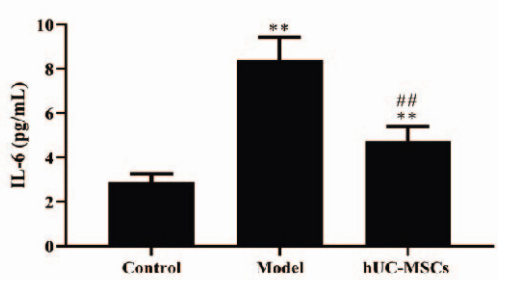

Fig. 3. hUC-MSCs reduced the level of proinflammatory cytokines in femoral heads. The serum level of TNF- $\alpha$ (A), IL-1 $\beta$ (B) and IL-6 (C) was determined by ELISA kits. The means ${ }_{ \pm} \mathrm{SD}$ of six independent samples were shown. ${ }^{* *} \mathrm{p}<0.01$, compared with control group; $\# \mathrm{p}<0.01$, compared with model group.

enhanced, and hUC-MSCs decreased the expression of proinflammatory cytokines in the femoral heads of GC-induced ONFH rat model.
hUC-MSCs inhibited the AKT/mTOR signaling pathway

It has been demonstrated that the AKT/mTOR signaling pathway is closely associated with the M1/M2 polar- 
A

Control Model hUC-MSCs

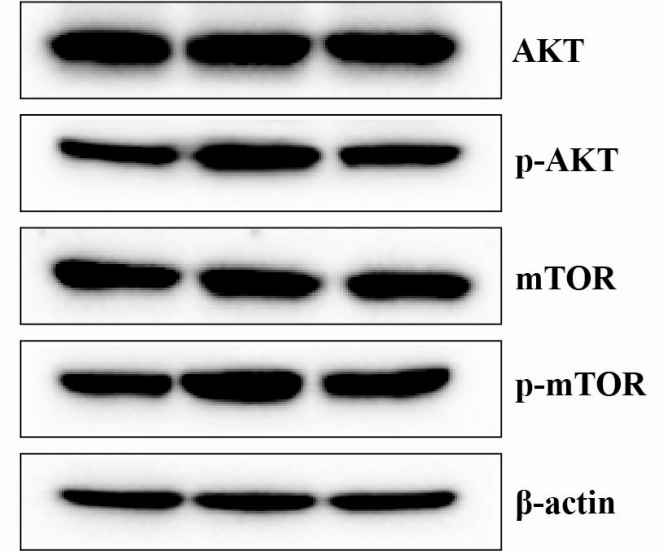

B

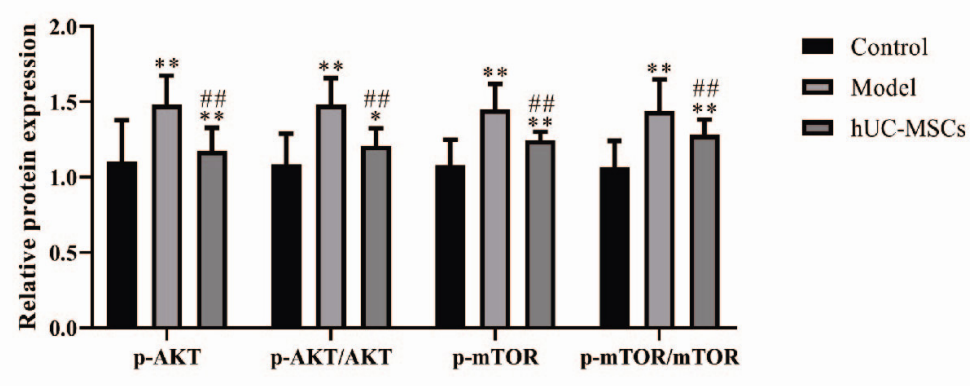

Fig. 4. hUC-MSCs repressed the activation of $A K T / m T O R$ signaling pathway. The protein expression of AKT, mTOR, p-AKT and p-mTOR was detected using western blot. The data were expressed after being normalized to $\beta$-actin. The means $\pm \mathrm{SD}$ of six independent samples were shown. ${ }^{*} \mathrm{p}<0.05$ and ${ }^{* *} \mathrm{p}<0.01$, compared with control group; ${ }^{\#} \mathrm{p}<0.01$, compared with model group.

ization (18). As illustrated in Fig. 4, the protein expression of phosphorylated AKT and mTOR, the radio of $\mathrm{p}-\mathrm{AKT}$ to AKT and the radio of $\mathrm{p}-\mathrm{mTOR}$ to $\mathrm{mTOR}$ were observably increased in the model group compared with that in the control group, which was prominently antagonized with hUC-MSCs treatment. The results suggested that hUC-MSCs suppressed the activation of AKT/mTOR signaling pathway.

\section{Discussion}

In the present study, hUC-MSCs reduce the necrosis and apoptosis of the femoral head, the macrophage polarization, the level of TNF- $\alpha$, IL- $1 \beta$ and IL- 6 in the femoral heads, and the protein expression of $\mathrm{p}-\mathrm{AKT}$ and p-mTOR in vivo. Therefore, hUC-MSCs may prevent necrosis and apoptosis through reducing $\mathrm{Ml}$ infiltration in the GC-induced ONFH model, which is associated with the $\mathrm{AKT} / \mathrm{mTOR}$ signaling pathway.

GC-induced ONFH has multiple and complicated pathogenesis including apoptosis, oxidative stress, aberrant lipid metabolism and vasospasm (19-21). Among them, apoptosis is an outstanding determinant of GC-induced ONFH. Previous studies have reported that GC induces ONFH through osteoblast apoptosis (22, 23). Similarly, our results showed that the osteocyte apoptosis was notably increased in a GC-induced ONFH rat model, which revealed the vital role of apoptosis in ONFH. In addition, hUC-MSCs have exhibited the anti-apoptotic effect in a variety of disease models. For instance, hUC-MSCs were effective for acute optic nerve injury in the early time through the anti-apoptotic, anti-inflammatory and proneuroregenerative roles (24). Ma et al. (14) showed that hUC-MSCs could reduce the TUNEL-positive cells in traumatic brain injury model. Corresponding to these findings, our results also revealed that hUC-MSCs observably reversed the osteocyte apoptosis of GC-induced ONFH rat model, which indicated hUC-MSCs could play an anti-apoptotic role in GC-induced ONFH model. Moreover, hUC-MSCs alleviated the pathological necrosis shown in the model group, which suggested that hUCMSCs could prevent necrosis in the GC-induced ONFH model. Taken together, hUC-MSCs may prevent necrosis and osteocyte apoptosis in the GC-induced ONFH model.

Glucocorticoids play an important role in immune regulation. On the one hand, glucocorticoids activate and strengthen the innate immune system, thus promoting inflammation. On the other hand, glucocorticoids inhibit the adaptive immune system to help homeostasis, thereby taking an anti-inflammatory effect (25). At the early stage of GC-induced ONFH, abnormal activation and infiltration of proinflammatory macrophages were identified in the necrotic sites $(26,27)$. As CD86 and CD206 are representative markers of $\mathrm{M} 1$ and M2 phenotype (8), the average optical density of CD86 and CD206 can reflect the number of $\mathrm{M} 1$ and M2 macrophage. F4/80 is mainly expressed on the surface of macrophages and used as a marker for mature macrophage (28). Thus, in the present study, the M1 and M2-type macrophages were increased and decreased in a GC-induced $\mathrm{ONFH}$ rat model, respectively. Meanwhile, hUC-MSCs antagonized the change of CD86 and CD206 of model group, which in- 
dicated that hUC-MSCs reduced the polarization of M1type macrophages in the GC-induced ONFH model. hUCMSCs have demonstrated the macrophage polarization effects in type 2 diabetes (29) and Sjögren's syndrome dry eye (30) disease models. Therefore, in line with these previous findings, our results indicated that hUC-MSCs could reduce the polarization of M1-type macrophages in a GC-induced ONFH rat model.

$\mathrm{AKT} / \mathrm{mTOR}$ signaling pathway is involved in various biological processes including cell proliferation, cell cycle and protein synthesis $(31,32)$. AKT/mTOR signaling pathway has been reported to regulate the macrophage polarization $(33,34)$. Furthermore, Zhao et al. (35) showed that pinocembrin relieved GC-induced apoptosis through inhibiting the PI3K/AKT/mTOR signaling pathway in osteocytes. Tang et al. (36) reveals that miR-27a mitigates $\mathrm{ONFH}$ via modulating the $\mathrm{PI} 3 \mathrm{~K} / \mathrm{AKT} / \mathrm{mTOR}$ signaling pathway. In the present study, the results showed an increase of phosphorylation AKT and mTOR, which inverted with hUC-MSCs treatment. Taken together, $\mathrm{AKT} / \mathrm{mTOR}$ signaling pathway was upregulated in the GC-induced ONFH model, which could be antagonized with hUC-MSCs treatment.

In summary, in vivo studies revealed that hUC-MSCs improved the necrosis and osteocyte apoptosis in GC-induced ONFH model through reducing the macrophage polarization, which was associated with the inhibition of AKT/mTOR signaling pathway. However, there are some limitations in the present study. For instance, although the TUNNEL results showed that apoptosis notably present in the model rats, the specific cell types really that underwent apoptosis did not indicate definitely. Thus, co-staining of osteocyte markers and TUNEL would be performed in future research. In brief, we hope our study can lay a firm basis for the prevention and treatment of GC-induced ONFH.

\section{Acknowledgments}

This work was supported by Key R\&D Projects of Shandong Province (2019GSF107007), and Special Fund for Taishan Scholars Project (TS201511110).

\section{Potential Conflict of Interest}

The authors have no conflicting financial interest.

\section{Availability of Data and Materials}

The datasets used or analyzed during the current study are available from the corresponding author.

\section{Supplementary Materials}

Supplementary data including one figure can be found with this article online at https://doi.org/10.15283/ijsc21120.

\section{References}

1. Zhao D, Zhang F, Wang B, Liu B, Li L, Kim SY, Goodman SB, Hernigou P, Cui Q, Lineaweaver WC, Xu J, Drescher WR, Qin L. Guidelines for clinical diagnosis and treatment of osteonecrosis of the femoral head in adults (2019 version). J Orthop Translat 2020;21:100-110

2. Tan G, Kang PD, Pei FX. Glucocorticoids affect the metabolism of bone marrow stromal cells and lead to osteonecrosis of the femoral head: a review. Chin Med J (Engl) 2012;125:134-139

3. Seguro LP, Rosario C, Shoenfeld Y. Long-term complications of past glucocorticoid use. Autoimmun Rev 2013;12: 629-632

4. Pan TT, Pan F, Gao W, Hu SS, Wang D. Involvement of macrophages and spinal microglia in osteoarthritis pain. Curr Rheumatol Rep 2021;23:29

5. Yunna C, Mengru H, Lei W, Weidong C. Macrophage M1/M2 polarization. Eur J Pharmacol 2020;877:173090

6. Chen G, Ni Y, Nagata N, Zhuge F, Xu L, Nagashimada M, Yamamoto S, Ushida Y, Fuke N, Suganuma H, Kaneko S, Ota T. Lycopene alleviates obesity-induced inflammation and insulin resistance by regulating $\mathrm{M1} / \mathrm{M} 2$ status of macrophages. Mol Nutr Food Res 2019;63:e1900602

7. Zhou X, Li W, Wang S, Zhang P, Wang Q, Xiao J, Zhang C, Zheng X, Xu X, Xue S, Hui L, Ji H, Wei B, Wang H. YAP aggravates inflammatory bowel disease by regulating M1/M2 macrophage polarization and gut microbial homeostasis. Cell Rep 2019;27:1176-1189.e5

8. Chen S, Lu Z, Wang F, Wang Y. Cathelicidin-WA polarizes E. coli K88-induced M1 macrophage to M2-like macrophage in RAW264.7 cells. Int Immunopharmacol 2018;54: $52-59$

9. Mathis D. Immunological goings-on in visceral adipose tissue. Cell Metab 2013;17:851-859

10. Weinstein RS, Nicholas RW, Manolagas SC. Apoptosis of osteocytes in glucocorticoid-induced osteonecrosis of the hip. J Clin Endocrinol Metab 2000;85:2907-2912

11. Calder JD, Buttery L, Revell PA, Pearse M, Polak JM. Apoptosis--a significant cause of bone cell death in osteonecrosis of the femoral head. J Bone Joint Surg Br 2004;86: 1209-1213

12. Lim JY, Jeong CH, Jun JA, Kim SM, Ryu CH, Hou Y, Oh W, Chang JW, Jeun SS. Therapeutic effects of human umbilical cord blood-derived mesenchymal stem cells after intrathecal administration by lumbar puncture in a rat model of cerebral ischemia. Stem Cell Res Ther 2011;2:38

13. Yang C, Wang G, Ma F, Yu B, Chen F, Yang J, Feng J, Wang Q. Repeated injections of human umbilical cord blood-derived mesenchymal stem cells significantly pro- 
motes functional recovery in rabbits with spinal cord injury of two noncontinuous segments. Stem Cell Res Ther 2018; 9:136

14. Ma J, Liu N, Yi B, Zhang X, Gao BB, Zhang Y, Xu R, Li X, Dai Y. Transplanted hUCB-MSCs migrated to the damaged area by SDF-1/CXCR4 signaling to promote functional recovery after traumatic brain injury in rats. Neurol Res 2015;37:50-56

15. Peng Y, Pan W, Ou Y, Xu W, Kaelber S, Borlongan CV, Sun $M, Y u$ G. Extracardiac-lodged mesenchymal stromal cells propel an inflammatory response against myocardial infarction via paracrine effects. Cell Transplant 2016;25: 929-935

16. Bernardo ME, Fibbe WE. Mesenchymal stromal cells: sensors and switchers of inflammation. Cell Stem Cell 2013;13: 392-402

17. Peng Y, Chen B, Zhao J, Peng Z, Xu W, Yu G. Effect of intravenous transplantation of hUCB-MSCs on M1/M2 subtype conversion in monocyte/macrophages of AMI mice. Biomed Pharmacother 2019;111:624-630

18. Vergadi E, Ieronymaki E, Lyroni K, Vaporidi K, Tsatsanis C. Akt signaling pathway in macrophage activation and M1/M2 polarization. J Immunol 2017;198:1006-1014

19. Weinstein RS. Glucocorticoids, osteocytes, and skeletal fragility: the role of bone vascularity. Bone 2010;46:564-570

20. Mont MA, Cherian JJ, Sierra RJ, Jones LC, Lieberman JR. Nontraumatic osteonecrosis of the femoral head: where do we stand today? A ten-year update. J Bone Joint Surg Am 2015;97:1604-1627

21. Mankin HJ. Nontraumatic necrosis of bone (osteonecrosis). N Engl J Med 1992;326:1473-1479

22. Nie Z, Chen S, Peng H. Glucocorticoid induces osteonecrosis of the femoral head in rats through GSK3 $\beta$-mediated osteoblast apoptosis. Biochem Biophys Res Commun 2019; 511:693-699

23. Peng P, Nie Z, Sun F, Peng H. Glucocorticoids induce femoral head necrosis in rats through the ROS/JNK/c-Jun pathway. FEBS Open Bio 2021;11:312-321

24. Chen M, Xiang Z, Cai J. The anti-apoptotic and neuro-protective effects of human umbilical cord blood mesenchymal stem cells (hUCB-MSCs) on acute optic nerve injury is transient. Brain Res 2013;1532:63-75

25. Busillo JM, Cidlowski JA. The five Rs of glucocorticoid action during inflammation: ready, reinforce, repress, resolve, and restore. Trends Endocrinol Metab 2013;24:109-119

26. Wu X, Feng X, He Y, Gao Y, Yang S, Shao Z, Yang C, Wang H, Ye Z. IL-4 administration exerts preventive effects via suppression of underlying inflammation and TNF- $\alpha$-induced apoptosis in steroid-induced osteonecrosis. Osteoporos Int 2016;27:1827-1837

27. Okazaki S, Nishitani Y, Nagoya S, Kaya M, Yamashita T, Matsumoto H. Femoral head osteonecrosis can be caused by disruption of the systemic immune response via the tolllike receptor 4 signalling pathway. Rheumatology (Oxford) 2009;48:227-232

28. Austyn JM, Gordon S. F4/80, a monoclonal antibody directed specifically against the mouse macrophage. Eur J Immunol 1981;11:805-815

29. Yin Y, Hao H, Cheng Y, Zang L, Liu J, Gao J, Xue J, Xie Z, Zhang Q, Han W, Mu Y. Human umbilical cord-derived mesenchymal stem cells direct macrophage polarization to alleviate pancreatic islets dysfunction in type 2 diabetic mice. Cell Death Dis 2018;9:760

30. Lu X, Li N, Zhao L, Guo D, Yi H, Yang L, Liu X, Sun D, Nian H, Wei R. Human umbilical cord mesenchymal stem cells alleviate ongoing autoimmune dacryoadenitis in rabbits via polarizing macrophages into an anti-inflammatory phenotype. Exp Eye Res 2020;191:107905

31. Ma C, Zhu L, Wang J, He H, Chang X, Gao J, Shumin W, Yan T. Anti-inflammatory effects of water extract of Taraxacum mongolicum hand.-Mazz on lipopolysaccharide-induced inflammation in acute lung injury by suppressing $\mathrm{PI} 3 \mathrm{~K} / \mathrm{Akt} / \mathrm{mTOR}$ signaling pathway. J Ethnopharmacol 2015;168:349-355

32. Banerjee N, Kim H, Talcott S, Mertens-Talcott S. Pomegranate polyphenolics suppressed azoxymethane-induced colorectal aberrant crypt foci and inflammation: possible role of miR-126/VCAM-1 and miR-126/PI3K/AKT/mTOR. Carcinogenesis 2013;34:2814-2822

33. Zhou J, Zhang A, Fan L. HSPA12B secreted by tumor-associated endothelial cells might induce $\mathrm{M} 2$ polarization of macrophages via activating $\mathrm{PI} 3 \mathrm{~K} / \mathrm{Akt} / \mathrm{mTOR}$ signaling. Onco Targets Ther 2020;13:9103-9111

34. Wei Y, Liang M, Xiong L, Su N, Gao X, Jiang Z. PD-L1 induces macrophage polarization toward the M2 phenotype via Erk/Akt/mTOR. Exp Cell Res 2021;402:112575

35. Zhao F, Qin Y, Yang J, Liu P, He X, Zhou L, Zhou S, Gui L, Zhang H, Wang X, Jiang S, Zhong Q, Zhou Y, Shi $\mathrm{Y}$. R-CHOP immunochemotherapy plus surgery is associated with a superior prognosis in Chinese primary intestinal diffuse large B-cell lymphoma. Asia Pac J Clin Oncol 2020;16:385-391

36. Tang J, Yu H, Wang Y, Duan G, Wang B, Li W, Zhu Z. miR-27a promotes osteogenic differentiation in glucocorticoid-treated human bone marrow mesenchymal stem cells by targeting PI3K. J Mol Histol 2021;52:279-288 